\title{
Due tavolette neo-sumeriche di ricevute di orzo e di argento
}

\author{
Francesco POMPONIO ${ }^{*}$ \\ Università di Messina
}

La collezione di tavolette neo-sumeriche del British Museum numerata 1923-1-13 è costituita da più di 250 tavolette inedite da Drehem, la più ricca collezione di testi da questo sito conservata nel BM, come ci informa il Dr. C. B. F. Walker. Pressoché la totalità di queste tavolette, tutte di piccole dimensioni, riguarda bovini, ovini, capridi, equidi e orsi, come è norma per la documentazione di Drehem. Fanno eccezione due testi, BM 115815 (dimensioni: 32×34

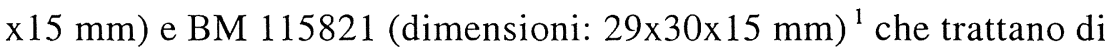
orzo e argento ${ }^{2}$ :

*nabium@virgilio.it.

1 Le tavolette sono pubblicate per gentile concessione del Dr. Walker e dei Trustees del British Museum. I testi sono stati traslitterati durante una missione al BM compiuta nell'ambito della ricerca PRIN 2002-2004 dal titolo di «Aspetti amministrativi ed economici della gestione dell'argento e degli altri beni pregiati nella documentazione paleo-accadica e neo-sumerica».

2 Inoltre, della medesima collezione fa parte una tavoletta di Sîn-kašid (BM 115800; 1923-1-13-18) del tutto identica, ad es., a quella edita in MVN 1037 (per questo testo, cf. da ultimo R. D. FRAYNE, RIME 4, pp. 444-447, N. 3), e naturalmente proveniente da Uruk: recto: ${ }^{\mathrm{d} E N-Z U-k a ̀-s ̌ i-i d ~ / ~ n i t a-k a l-g a ~ / ~ l u g a l-u n u g k i ~}{ }^{\mathrm{ki}}$ a / ú-a / é-an-na; verso: é-gal / nam-lugal-la/-ka-ni / mu-dù.

Sefarad 64 (2004) págs. 397-407

(c) CSIC

ISSN 037-0894 


\section{BM 115815}

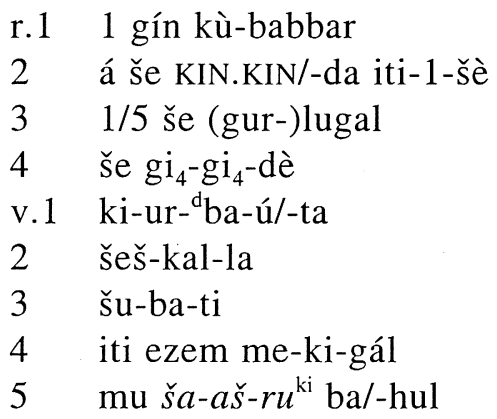

«1 siclo di argento per il lavoro di mietitura di 1 mese; 60 sìla di orzo da restituire. Da Ur-Bau Šeškalla ha ricevuto.

Mese della festa di Mekigal. Anno in cui Šašru fu razziata».

\section{BM 115821}

r.1 1/5 4/30 5 sìla še (gur-)lugal

2 kù-bi $1 / 2$ gín

3 ki-ur- ${ }^{\mathrm{d}} \mathrm{ba}-\mathrm{u}-\mathrm{ta}$

4 šeš-kal-la

5 šu-ba-ti

v. spazio anepigrafo

1 iti me-igi-gál

2 mu en- ${ }^{\mathrm{d}}$ nanna / máš-e ì-pà

«105 sila di orzo secondo il (gur) reale; suo equivalente in argento: $1 / 2$ siclo.

Da Ur-Bau Šeškalla ha ricevuto.

Mese di Megigal. Anno in cui la somma sacerdotessa di Nanna fu scelta mediante il capretto.»

Le due tavolette sono datate al medesimo mese del Reichskalender. La grafia ortografica del nome del dio Mekigal (me-igi-gál) impiegata nel nome del mese di BM 115821:v.1 ricorre in qualche altro testo di Drehem (TCL 2 5503:v.II.10; 5524:v.5; TIM 6 27:v.5). 
Sef 64:2 (2004) DUE TAVOLETTE NEO-SUMERICHE DI RICEVUTE DI ORZO E DI ARGENTO 399

Tavolette di Drehem che registrano la ricevuta di un'unica quantità di orzo non sono frequenti: in alcuni testi questo orzo è messo in relazione con l'allevamento del bestiame, come, ad es., SAT 2 518 , dove 30 gur di orzo, definiti šà-gal udu-niga urim ${ }_{5}{ }^{\mathrm{ki}}$-ma, «per l'alimentazione di pecore ingrassate in Ur», sono forniti da Atu al kurušda $\mathrm{Nalu}^{3}$. In altri testi, l'orzo è in quantità molto più ridotta $\mathrm{e}$ riguarda personaggi che non sembrano far parte del personale amministrativo di Drehem. H. Lutzmann ${ }^{4}$ elenca alcuni contratti di prestito di orzo che sono assegnati a Drehem, ma apparentemente solo sulla base dell'impiego del Reichskalender. A questi sarebbero da aggiungere, a motivo dello stesso elemento, i testi di orzo NATN $257,308,326,336,342,524$ e 760 e quelli di argento NATN 16, 422 e 847.

La prima delle due tavolette del Britih Museum registra la consegna da parte di Ur-Bau a Šeškalla di 1 siclo d'argento per il futuro lavoro di questi come mietitore per la durata di un mese e, in più, di 60 sìla di orzo, da intendere come un prestito considerando che è precisato che essi devono essere restituiti. Poiché la normale paga di un mietitore ingaggiato nel periodo di Ur III è di 6 sìla di orzo, in ambito istituzionale, sulla base dell'equivalenza 1 siclo $=$ 300 sìla, Šeškalla viene a ricevere la paga, notevolmente superiore, di 10 sìla al giorno, come del resto è prescritto in qualche altro ingaggio di mietitori da parte di privati ${ }^{5}$. Il fatto che, al contempo, egli prenda in prestito 60 sìla di orzo dal suo futuro datore di lavoro, da un canto dimostra che il pagamento dell'attività di mietitore avveniva a lavoro compiuto, dall'altro che Ur-Bau poteva contare su una pronta restituzione sia della quantità di orzo prestata, sia del suo interesse, in tutto 80 sìla (considerando l'ammontare del prestito di orzo al 33\%): infatti, da lì a un mese il suo debitore

\footnotetext{
3 Il sigillo impresso sulla tavoletta è di un terzo funzionario, Lani(mu), ed è indicato sulla tavoletta come kišib lá-ni-mu šà unug ${ }^{\text {ki }}$-ga.

4 H. Lutzmann, Die neusumerischen Schuldurkunden (Heidelberg 1976) p. 98.

5 Cf. P. Steinkeller, «Money-Lending Practices in Ur III Babylonia: The Issue of Economic Motivation», in Debt and Economic Renewal in the Ancient Near East. A Colloquium Held at Columbia University (November 1988), vol. III, eds. M. Hudson and M. van de Mieroop (Bethesda 2002) p. 129, n. 8.
} 
avrebbe avuto a disposizione una somma di argento che gli avrebbe procurato circa 300 sìla di orzo. Su questo punto torneremo più sotto.

Anche la seconda tavoletta deve essere interpretata come riferita a un prestito, questa volta di 105 sìla di orzo, che intercorre tra $\mathrm{i}$ medesimi personaggi ${ }^{6}$. In BM 115821, come in BM 115815, mancano alcune caratteristiche essenziali dei contratti di prestito, in particolare la lista dei testimoni, poiché l'indicazione dell'ammontare del prestito e il giuramento in nome del re possono non essere indicati e l'impronta del sigillo potrebbe comparire sulla custodia, perduta, della tavoletta; quindi, il nostro testo non può essere propriamente considerato un contratto. Nel documento è precisato l'equivalente dell'orzo in argento, ed è da notare che nella tavoletta, datata alla fine dell'anno, alla pari dell'altra, e, quindi, prima della mietitura, il valore dell'orzo in argento è insolitamente alto: in luogo del corrispettivo normale 300 sìla di orzo $=1$ siclo di argento, abbiamo 105 sìla $=1 / 2$ siclo, cioè 210 sìla $=1$ siclo. La precisazione dell'equivalente dell'orzo in argento e il valore eccezionalmente elevato del primo potrebbero indicare la volontà del creditore di premunirsi contro la semplice restituzione della quantità di orzo ceduta, cioè l'intenzione di ricavare 200 sìla ( $=150$ sìla, la normale quantità di orzo per $1 / 2$ siclo di argento +50 sìla, il suo interesse al $33 \%$ ) dai 105 effettivamente versati qualche mese prima.

A motivo della somiglianza dei due testi, in primo luogo l'identità dei due personaggi citati, è da supporre che i due testi siano vicini tra loro nel tempo. Ma entrambi i nomi di anno hanno due possibili interpretazioni: l'anno della scelta dell'alta sacerdotessa di Nanna può essere Š 43 o AS 4, quello della razzia di Šašru può essere $\breve{S} 42$ o AS 6. In ogni caso, tra i due testi ci sono un anno o

6 Un Ur-Bau è citato spesso nella documentazione di Drehem come fornitore di bestiame (ki-...-ta) e come maškim e uno Šeškalla come ricevitore (ì-dab ${ }_{5}$ ) di bestiame, ma i due funzionari non sono mai menzionati insieme. Data l'attività di mietitore di Šeškalla in BM 115815, è da escludere che questi funzionari di Drehem, dai nomi così frequenti, siano gli stessi personaggi menzionati nelle nostre due tavolette. 
due di differenza. Ma il rapporto tra questi due personaggi sembra essersi prolungato nel tempo: vi è un testo datato addirittura a ŠS 8 $(S A T 31862)^{7}$, e questo suggerirebbe per le nostre due tavolette la datazione più recente (AS 4-6), che pure registra una quantità di orzo (appena 30 sìla), che Šeškalla ha ricevuto (šu-ba-an-ti) da UrBau. Anche quest' orzo deve essere restituito ( $\mathrm{gi}_{4}-\mathrm{gi}_{4}$-dam) e, quindi, anche in questo caso deve trattarsi della registrazione di un prestito. Il mese di redazione di quest'ultimo testo è iti ezem- ${ }^{\mathrm{d}} \mathrm{Nin}-\mathrm{a}-\mathrm{zu}$, cioè il VI mese del Reichskalender. Un altro elemento interessante di SAT 31862 è la presenza dell'impronta del sigillo: ur- ${ }^{\mathrm{d}}$ ba'-ú / dumu du-du, che, come si vede, eccezionalmente appartiene al personaggio che fornisce, non a quello che riceve l'orzo.

Ora, questo piccolo archivio deve essere aggiunto all'archivio di Ur-Bau, studiato da ultimo da P. Steinkeller ${ }^{8}$, che lo assegna, sulla base dell'onomastica e della toponomastica alla provincia di Lagaš, o meno verosimilmente a quella di Umma, mentre secondo $\mathrm{H}$. Neumann ${ }^{9}$, l'archivio potrebbe provenire da un centro della provincia di Nippur.

Il cosiddetto dossier di Ur-Bau, finora datato da $\breve{S} 40$ ad AS 1, con l'aggiunta di SAT 31862 arriva fino all'anno ŠS 8, e si estende, quindi, per più di un quarto di secolo. Esso è, dunque, allo stato attuale della nostra documentazione, non dissimile per durata da quello ben più numeroso (76 testi) del più celebre SI:A-a, che va da $\breve{S} 40$ a IS $2{ }^{10}$. L'archivio di Ur-Bau comprende undici testi ${ }^{11}$ : uno

7 Il dr. Manuel Molina, che cordialmente ringrazio, mi ha segnalato il rapporto delle due tavolette del BM con questo testo, oltre a fornire molti suggerimenti nella stesura dell'articolo.

8 P. STEINKeller, «Money-Lending Practices...», pp. 120-121.

9 H. NeumanN, «Zum Problem der privaten Feldpacht in neusumerischer Zeit», in Šlmu IV. Everyday life in Ancient Near East: Papers Presented at the International Conference (Poznan, 19-22 September, 1989), eds. J. ZABLOCKA and S. ZAWADZKI (Poznan 1993) p. 231.

${ }^{10}$ Cf. da ultimo S. GARFINKLE, «SI.A-a and His Family: the Archive of a 21 st Century (BC) Entrepreneur», ZA 93 (2003) pp. 161-198.

1 Ai dieci raccolti e studiati da P. STEINKELLER, «Money-Lending Practices...», pp. 120-121, è da aggiungere la tavoletta edita da M. HILGERT, Drehem Adminis- 
(YOS 4 30: AS 1/iti me-ki-gál) documenta l'ingaggio come mietitore per la durata di un mese di un certo Šahgi, con il pagamento di 1 siclo di argento, cioè alle identiche condizioni di Šeškalla nel nostro BM $115815^{12}$. Le altre dieci tavolette registrano la cessione di argento (in quattro casi) o di orzo (in sei) da parte di Ur-Bau a vari individui, alcuni dei quali ricorrono in più di un documento. $\mathrm{Nel}$ caso di cessione di orzo è impiegata regolarmente la formula še-ur ${ }_{5}$-ra máš gá-gá-dam, «orzo in prestito; l'interesse deve essere aggiunto»; in due testi dell'argento (PDT 1 631; OIP 115 467) è impiegata la stessa formula di BM 115815 (ma riguardo all'orzo): $\mathrm{gi}_{4}$-gi $\mathrm{g}_{4}$-dam, «deve essere restituito», in un altro dell'argento (SAT 2 262) la stessa formula usata nel medesimo archivio per l'orzo: kùur $_{5}$-ra maš gá-gá-dè, mentre nel quarto (YOS 421 ) manca qualsiasi riferimento all'interesse del debito o alla sua restituzione, ma è aggiunta la formula del giuramento (mu-lugal-bi in-pà).

Quel che è più interessante è che, in questi documenti di prestito, ben quattro dei debitori sembrano cedere a Ur-Bau per la coltivazione (apin-lá-šè) il proprio allotment field (šuku). Il problema che è stato posto a proposito di questi testi è se i terreni in questione (la cui estensione varia da 3 a $72 \mathrm{iku}$ ) fossero ceduti in un vero prestito anticretico, cioè perché al tempo stesso servissero da garanzia della restituzione del debito e il loro raccolto ne sostituisse l'interesse, o se il creditore stesse cercando, con buone prospettive di successo, di impadronirsi di essi. P. Steinkeller ${ }^{13}$ si pronunzia a favore della seconda ipotesi, che conviene alla sua ricostruzione che in Babilo-

trative Documents from the Reign of Šulgi, OIP 115 (Chicago 1998) no. 467. Il documento in questione, come le nostre due tavolette del BM, appartiene a un lotto, dell'Oriental Institute Museum di Chicago, che comprende quasi esclusivamente testi di Drehem (cf. ibid., p. 2, Accession Number 487: OIM A4126-A5997). Con la tavoletta di Chicago salgono a quattro i testi che registrano prestiti di UrBau ad Ammamu nell' arco di pochi mesi, e due, rispettivamente di 5 e 1 siclo di argento, nel medesimo mese (Š 43 X: YOS 421 e OIP 115 467).

${ }^{12}$ La terminologia è, però, diversa: á še-KIN 1 guruš $u_{4}-30$-šè (r. 2-3) e in più è menzionata la formula del giuramento: mu-lugal-bi in-pà (v. 3), in riferimento, con ogni probabilità, all'impegno del lavoratore di presentarsi al servizio.

13 P. Steinkeller, «The Ur III Period», in Security for Debt in Ancient Near Eastern Law, Culture and History of the Ancient Near East 9, eds. R. WestBroOK and R. JASNOW (Leiden 2001) p. 48. 
nia «most loans were made with objectives other than interest-generated profit in mind. As the extant data demonstrate clearly, the lender's primary objective in advancing loans was to get possession of either the borrower's labor or his land or often both» ${ }^{14}$.

Tuttavia, a questo riguardo, alcuni elementi andrebbero considerati: innanzitutto, i campi sui quali si sarebbe stesa l'avida mano di Ur-Bau sono campi-šuku, che non sembra possibile fossero alienati: a prescindere da ogni considerazione legale, essendo i campi-šuku forniti dall'amministrazione centrale o provinciale in cambio di servizi, si porrebbe la questione di chi avrebbe dovuto continuare ad assolvere questi servizi, a parte la lavorazione agricola del campo in oggetto: il precedente assegnatario del campo, che però per tutta la vita non avrebbe più ricavato dal suo lavoro, per la corona o per la provincia, neanche un sìla di orzo di quel campo, o Ur-Bau, che certo non avrebbe potuto svolgere contemporaneamente l'attività di Adada, Ammamu, Ur-Hendursag e Ur-Ninazu, per citare solo alcuni dei debitori dei quali terreni egli sarebbe riuscito a impadronirsi. Inoltre, i testi parlano di cessione del campo apin-lá-šè, «per la coltivazione», un termine che se ben conviene alla coltivazione per uno o più anni, perché il raccolto del campo sostituisse l'interesse non versato, non sembra addirsi a un passaggio di proprietà/possesso. Infine, a riguardo di BM 115815, abbiamo presentato un caso in cui il prestito di Ur-Bau sicuramente non poteva avere lo scopo di porre il debitore in condizioni tanto disperate da dover alienare uno dei propri beni.

Resta il problema della sproporzione, in alcuni casi, tra il rendimento di un campo (ad es., 9 iku che produrrebbero 15 gur di orzo $=15$ sicli di argento) e l'ammontare dell'interesse del debito $(1,8$ sicli, interesse di un debito di 9 sicli), ma questo problema rimane, anche ammettendo che Ur-Bau prestasse argento e orzo per potersi impadronire dei terreni del debitore insolvente, cioè rimarrebbe l'enorme sproporzione tra il valore del campo e il capitale prestato.

14 Una critica di questa interpretazione è presentata da S. GARFINKLE, «Shepherds, Merchants, and Credit. Some Observations on Lending Practices in Ur III Mesopotamia», JESHO 47 (2004) pp. 6-9. 
La risposta di P. Steinkeller è che «in many of the transactions discussed here, the borrowers simply had no choice: almost certainly, because of their prior indebtedness to creditors, the latter were able to impose those particular conditions on them» ${ }^{15}$. Ma se c'erano questi precedenti, e non pagati debiti, come è ad esempio il caso accertato del debitore Ammamu nei confronti di Ur-Bau ${ }^{16}$, è possibile ammettere che il prodotto del campo, eccessivo se confrontato all'interesse del debito citato, in realtà coprisse anche quelli dei precedenti debiti non pagati, ancorché non citati nel documento redatto in riferimento all'ultimo debito. Non sembrano esservi, quindi, elementi sufficienti per considerare i prestiti di Ur-Bau come miranti essenzialmente ad altro scopo che a quello di investire il proprio capitale.

Infine, è da accennare all'impiego del sigillo sui documenti in oggetto. Sulle tavolette del dossier in due soli casi compare l'impronta del sigillo: il primo testo, come su visto, è SAT 3 1862, e il sigillo è, stranamente, quello di Ur-Bau, cioè del creditore; il secondo è $M V N 13674$ e il sigillo appartiene a un personaggio, il gu-zalá Urdu-Nanna, che non è menzionato nella tavoletta, e che comunque non è né il creditore (Ur-Bau), né il debitore (Lu-Nanna). Ma Urdu-Nanna compare in un altro documento di prestito dell' archivio di Ur-Bau (PDT 2 1282), dove è detto che il suo sigillo è impresso in luogo di quello del debitore Adada: mu-a-da-da-šè kišib urdú'nanna íb-ra. Ed è lo stesso Urdu-Nanna, che evidentemente agisce come garante, che pronunzia il giuramento di restituzione del debito ${ }^{17}$. Da ultimo, in PDT 2 1244, è menzionata l'impressione del sigillo di un personaggio, $\mathrm{X}$-zi, che sembra agire per conto dei due debitori.

\footnotetext{
15 P. Steinkeller, «The Ur III Period», p. 54.

16 Cf. P. Steinkeller, «Money-Lending Practices...», p. 120, con l'aggiunta di OIP 115467.

17 Nello stesso testo, in cui il debito di soli 1.620 sìla di orzo implica la cessione «in coltivazione» di ben $72 \mathrm{iku}$ di terreno, è citato anche il nome di un quarto personaggio, Allamu, che autorizza (inim) la transazione.
} 
Sef 64:2 (2004) DUE TAVOLETTE NEO-SUMERICHE DI RICEVUTE DI ORZO E DI ARGENTO 405

BM 115815
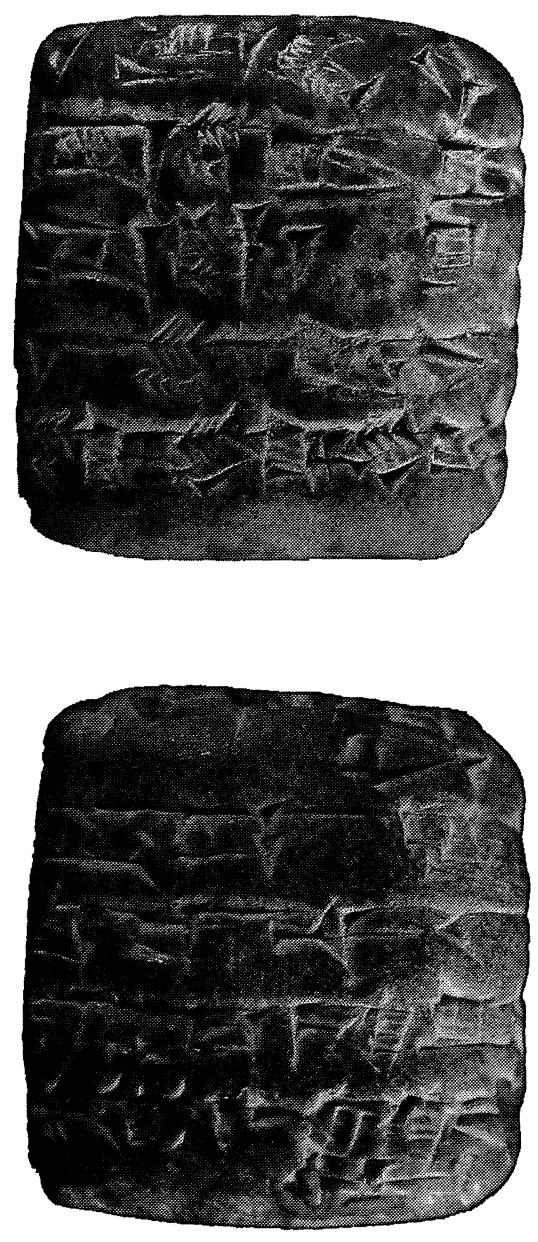
BM 115821
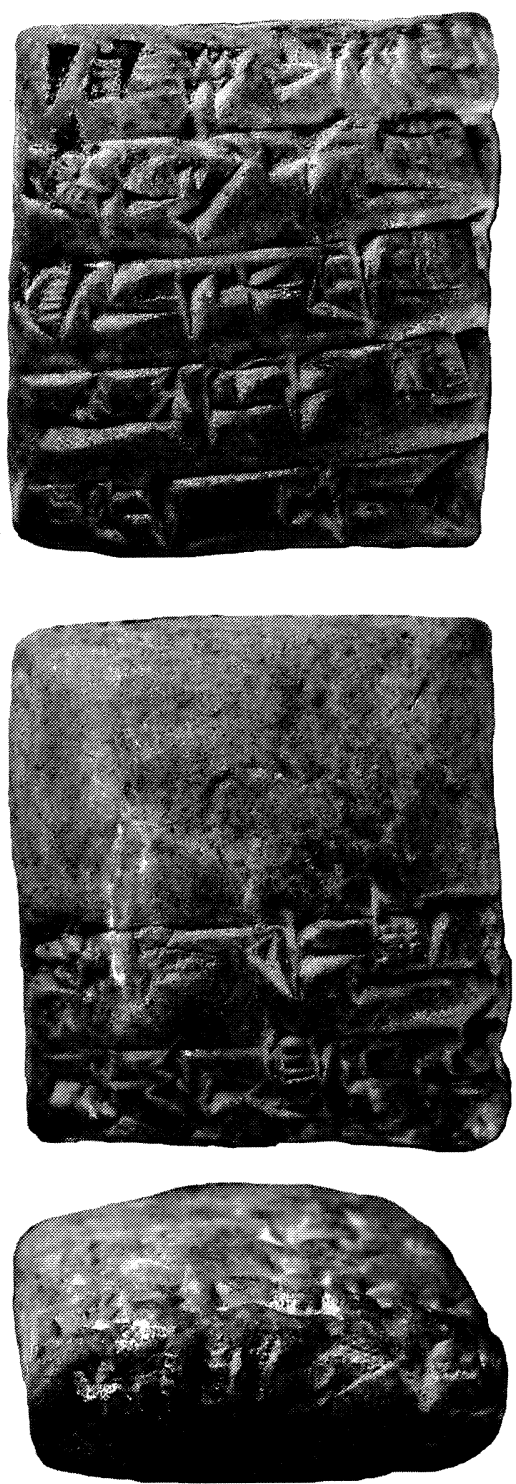
Sef 64:2 (2004) DUE TAVOLETTE NEO-SUMERICHE DI RICEVUTE DI ORZO E DI ARGENTO 407

\section{RESUMEN}

Los dos textos neo-sumerios del British Museum que aquí se editan se suman a los que conforman el dossier de documentos del prestamista Ur-Bau, de origen desconocido. En la primera tablilla, un tal Šeškalla recibe una cantidad de plata para un trabajo de recolección por un mes, además de una cantidad de cebada en préstamo. En la segunda, el mismo Šeškalla recibe de Ur-Bau una cantidad de cebada, también probablemente en este caso como préstamo, al poco habitual y elevado precio de 210 sìla por siclo de plata, en lugar del valor más normal de 300 sìla por siclo de plata.

PALABRAS ClAVE: Textos cuneiformes neo-sumerios, Nippur, préstamos.

\section{SUMMARY}

The two Neo-Sumerian texts from the British Museum published here have to be added to the dossier of Ur-Bau, a moneylender of unknown origin. In the first tablet a certain Šeškalla receives silver for one-month harvest work as well as barley on loan. In the second text, the same Šeškalla receives from Ur-Bau an amount of barley, in this case probably also on loan, at the unusually high value of 210 sila for 1 shekel of silver, the usual value being 300 sìla for 1 shekel.

KEYWORDS: Neo-Sumerian cuneiform texts, Nippur, loans. 